International Journal of Pure and Applied Mathematics

Volume 110 No. 1 2016, 103-124

ISSN: 1311-8080 (printed version); ISSN: 1314-3395 (on-line version)

url: http://www.ijpam.eu

doi: 10.12732/ijpam.v110i1.12

ijpam.eu

\title{
HOLDER AND INTEGRAL MEANS INEQUALITIES \\ FOR A CERTAIN SUBCLASS OF ANALYTIC FUNCTIONS \\ BASED ON CAPUTO'S FRACTIONAL OPERATOR
}

\author{
K. Thilagavathi \\ Department of Mathematics \\ School of Advanced Sciences \\ VIT University \\ Vellore, 632014, INDIA
}

\begin{abstract}
The main purpose of present paper is to investigate Holder and integral means inequalities for a certain subclass of analytic functions based on Caputo's fractional operator. Further we obtain the partial sum results for functions belonging to the new subclass. Moreover we point out some new or known consequences of our main result.
\end{abstract}

AMS Subject Classification: 30C45, 30C50

Key Words: analytic functions, starlike, convex, Hadamard product, Caputo's fractional operator

\section{Introduction}

Let $\mathcal{A}$ denote the class of functions of the form

$$
f(z)=z+\sum_{n=2}^{\infty} a_{n} z^{n}
$$

which are analytic and univalent in the open disc $\mathbf{U}=\{z: z \in \mathbf{C},|z|<1\}$. Also let $\mathcal{T}$ be a subclass of $\mathcal{A}$ consisting of functions of the form

$\begin{array}{lr}\text { Received: } & \text { July 14, } 2016 \\ \text { Revised: } & \text { August 30, } 2016 \\ \text { Published: } & \text { October 21, } 2016\end{array}$

(c) 2016 Academic Publications, Ltd. url: www.acadpubl.eu 


$$
f(z)=z-\sum_{n=2}^{\infty} a_{n} z^{n}, \quad a_{n} \geq 0, z \in \mathbf{U}
$$

We recall the following definitions due to owa [13].

Definition 1. Let the function $f(z)$ be analytic in a simply-connected region of the $z$ - plane containing the origin. The fractional integral of $f$ of order $\mu$ is defined by

$$
D_{z}^{-\mu} f(z)=\frac{1}{\Gamma(\mu)} \int_{0}^{z} \frac{f(\xi)}{(z-\xi)^{1-\mu}} d \xi, \quad \mu>0
$$

where the multiplicity of $(z-\xi)^{1-\mu}$ is removed by requiring $\log (z-\xi)$ to be real when $z-\xi>0$.

Definition 2. The fractional derivatives of order $\mu$, is defined for a function $f(z)$, by

$$
D_{z}^{\mu} f(z)=\frac{1}{\Gamma(1-\mu)} \frac{d}{d z} \int_{0}^{z} \frac{f(\xi)}{(z-\xi)^{\mu}} d \xi, \quad 0 \leq \mu<1,
$$

where the function $f(z)$ is constrained, and the multiplicity of the function $(z-\xi)^{-\mu}$ is removed as in Definition 1.

Definition 3. Under the hypothesis of Definition 2, the fractional derivative of order $n+\mu$ is defined by

$$
D_{z}^{n+\mu} f(z)=\frac{d^{n}}{d z^{n}} D_{z}^{\mu} f(z) \quad\left(0 \leq \mu<1, n \in \mathbf{N}_{0}\right) .
$$

With the aid of the above definitions and their known extensions involving fractional derivative and fractional integrals, the generalization of Salagean [16] derivative operator and Libera integral operator [8] was given by Owa[13]. Srivastava and owa [22] introduced the operator

$$
\Omega^{\delta}: \mathcal{A} \rightarrow \mathcal{A}
$$

defined by

$$
\Omega^{\delta} f(z)=\Gamma(2-\delta) z^{\delta} D_{z}^{\delta} f(z)=z+\sum_{n=2}^{\infty} \Phi(n, \delta) a_{n} z^{n}
$$


where

$$
\Phi(n, \delta)=\frac{\Gamma(n+1) \Gamma(2-\delta)}{\Gamma(n+1-\delta)} \text { and } \delta \in \mathbf{R}, \delta \neq 2,3,4 \ldots
$$

For $f \in \mathcal{A}$ and various choices of $\delta$, we get

$$
\begin{gathered}
\Omega^{0} f(z):=f(z)=z+\sum_{n=2}^{\infty} a_{n} z^{n}, \\
\Omega^{1} f(z):=z f^{\prime}(z)=z+\sum_{n=2}^{\infty} n a_{n} z^{n}, \\
\Omega^{j} f(z):=\Omega\left(\Omega^{j-1} f(z)\right)=z+\sum_{n=2}^{\infty} n^{j} a_{n} z^{n},(j=1,2,3, \ldots),
\end{gathered}
$$

which is known as Salagean operator (Salagean[16]). Also note that

$$
\Omega^{-1} f(z)=\frac{2}{z} \int_{0}^{z} f(t) d t:=z+\sum_{n=2}^{\infty}\left(\frac{2}{n+1}\right) a_{n} z^{n}
$$

and

$$
\Omega^{-j} f(z):=\Omega^{-1}\left(\Omega^{-j+1} f(z)\right):=z+\sum_{n=2}^{\infty}\left(\frac{2}{n+1}\right)^{j} a_{n} z^{n},(j=1,2,3, \ldots)
$$

called Libera integral operator[8]. We note that the Libera integral operator is generalized as Bernardi integral operator given by Bernardi [3] is

$$
\frac{1+\nu}{z^{\nu}} \int_{0}^{z} t^{\nu-1} f(t) d t:=z+\sum_{n=2}^{\infty}\left(\frac{1+\nu}{n+1}\right) a_{n} z^{n},(\nu=1,2,3, \ldots) .
$$

Further we recall the definition of the fractional-order derivative due to Caputos[4] given by

$$
D^{\alpha} f(t)=\frac{1}{\Gamma(n-\alpha)} \int_{a}^{t} \frac{f^{(n)}(\tau)}{(t-\tau)^{\alpha+1-n}}
$$

where $n-1<\Re(\alpha) \leq n, n \in \mathbf{N}$ and the parameter $\alpha$ is allowed to be real or even complex, $a$ is the initial value of the function $f$. 
Making use of these results and the definition of Caputo's fractional derivative [4], recently Salah and Darus in [17], introduced the following operator

$$
\mathbf{J}_{\mu}^{\eta} f(z)=\frac{\Gamma(2+\eta-\mu)}{\Gamma(\eta-\mu)} z^{\mu-\eta} \int_{0}^{z} \frac{\Omega^{\eta} f(t)}{(z-t)^{\mu+1-\eta}} d t
$$

where $\eta$ (real number) and $(\eta-1<\mu<\eta<2)$. That is

$$
\mathbf{J}_{\mu}^{\eta} f(z)=z+\sum_{n=2}^{\infty} \frac{(\Gamma(n+1))^{2} \Gamma(2+\eta-\mu) \Gamma(2-\eta)}{\Gamma(n+\eta-\mu+1) \Gamma(n-\eta+1)} a_{n} z^{n}, \quad(z \in \mathbf{U}) .
$$

We note that

$$
\mathbf{J}_{0}^{0} f(z)=f(z) \text { and } \mathbf{J}_{1}^{1} f(z)=z f^{\prime}(z)
$$

we making use of subclass $\mathcal{T} \mathbf{J}_{\mu}^{\eta}(\alpha, \beta, \gamma, A, B)$ of analytic functions with negative coefficients involving the operator $\mathbf{J}_{\mu}^{\eta}$ given by (14) and discuss some usual properties of the geometric function theory.

\section{The Class $\mathcal{T} \mathrm{J}_{\mu}^{\eta}(\alpha, \beta, \gamma, A, B)$}

Definition 4. For fixed $-1 \leq A \leq B \leq 1$ and $0<B \leq 1$, let $\mathcal{S} \mathbf{J}_{\mu}^{\eta}(\alpha, \beta, \gamma, A, B)$ denote the subclass of $\mathcal{A}$ consisting of functions $f$ of the form (1) and satisfying the condition

$$
\left|\frac{\frac{z\left(\mathbf{J}_{\mu}^{\eta} f(z)\right)^{\prime}}{\mathbf{J}_{\mu}^{\eta} f(z)}-1}{2 \gamma(B-A)\left(\frac{z\left(\mathbf{J}_{\mu}^{\eta} f(z)\right)^{\prime}}{\mathbf{J}_{\mu}^{\eta} f(z)}-\alpha\right)-B\left(\frac{z\left(\mathbf{J}_{\mu}^{\eta} f(z)\right)^{\prime}}{\mathbf{J}_{\mu}^{\eta} f(z)}-1\right)}\right|<\beta, \quad z \in \mathbf{U},
$$

where $\mathbf{J}_{\mu}^{\eta} f(z)$ is given by (14) and

$$
0 \leq \alpha<1, \quad 0<\beta \leq 1, \quad \frac{B}{2(B-A)}<\gamma \leq \begin{cases}\frac{B}{2(B-A) \alpha} & \alpha \neq 0 \\ 1 & \alpha=0\end{cases}
$$

We also let $\mathcal{T} \mathbf{J}_{\mu}^{\eta}(\alpha, \beta, \gamma, A, B)=\mathcal{S} \mathbf{J}_{\mu}^{\eta}(\alpha, \beta, \gamma, A, B) \cap \mathcal{T}$, where $\mathcal{T}$ is given by $(2)$

Remark 5. By suitably specializing the values of $A, B, \alpha, \beta$ and $\gamma$ the class $\mathcal{T} \mathbf{J}_{\mu}^{\eta}(\alpha, \beta, \gamma, A, B)$ leads to known subclasses studied in Aghalary and Kullkarni[1], Khairanar and Meena[7], Owa and Nishiwaki [12] and various new subclasses. 
For convenience in our study we consider $0 \leq \alpha<1,0<\beta \leq 1$,

$$
\frac{B}{2(B-A)}<\gamma \leq \begin{cases}\frac{B}{2(B-A) \alpha} & \alpha \neq 0 \\ 1 & \alpha=0\end{cases}
$$

for fixed $-1 \leq A \leq B \leq 1$ and $0<B \leq 1$. Further

$$
C_{n}(\eta, \mu)=\frac{(\Gamma(n+1))^{2} \Gamma(2+\eta-\mu) \Gamma(2-\eta)}{\Gamma(n+\eta-\mu+1) \Gamma(n-\eta+1)}
$$

and

$$
C_{2}(\eta, \mu)=\frac{4 \Gamma(2+\eta-\mu) \Gamma(2-\eta)}{\Gamma(3+\eta-\mu) \Gamma(1-\eta)} .
$$

Theorem 6. (Characterization property:) Let the function $f$ be defined by (2) is in the class $\mathcal{T} \mathbf{J}_{\mu}^{\eta}(\alpha, \beta, \gamma, A, B)$ if and only if

$$
\sum_{n=2}^{\infty}[2 \beta \gamma(B-A)(n-\alpha)+(1-B \beta)(n-1)] C_{n}(\eta, \mu)\left|a_{n}\right| \leq 2 \beta \gamma(1-\alpha)(B-A),
$$

where $C_{n}(\eta, \mu)$ is given by $(16)$.

Corollary 7. Let the function $f$ defined by (2) be in the class $\mathcal{T} \mathbf{J}_{\mu}^{\eta}(\alpha, \beta, \gamma$, $A, B)$, then we have

$$
\left|a_{n}\right| \leq \frac{2 \beta \gamma(1-\alpha)(B-A)}{[2 \beta \gamma(B-A)(n-\alpha)+(1-B \beta)(n-1)] C_{n}(\eta, \mu)}
$$

the equation (19) is attained for the function

$$
f(z)=z-\frac{2 \beta \gamma(1-\alpha)(B-A)}{[2 \beta \gamma(B-A)(n-\alpha)+(1-B \beta)(n-1)] C_{n}(\eta, \mu)} z^{n} \quad(n \geq 2)
$$

where $C_{n}(\eta, \mu)$ is given by (16).

For the sake of brevity, we let

$$
\Phi_{n}(\alpha, \beta, \gamma, A, B)=2 \beta \gamma(B-A)(n-\alpha)+(1-B \beta)(n-1)
$$

and

$$
\Phi_{2}(\alpha, \beta, \gamma, A, B)=1+2 \beta \gamma(B-A)(2-\alpha)-B \beta
$$


Theorem 8. (Extreme Points:) Let

$$
f_{1}(z)=z
$$

and

$$
f_{n}(z)=z-\frac{2 \beta \gamma(1-\alpha)(B-A)}{\Phi_{n}(\alpha, \beta, \gamma, A, B) C_{n}(\eta, \mu)} z^{n},(n \geq 2)
$$

where $C_{n}(\eta, \mu)$ is given by (16). Then $f \in \operatorname{athcalT} \mathbf{J}_{\mu}^{\eta}(\alpha, \beta, \gamma, A, B)$ if and only if it can be expressed in the form

$$
f(z)=\sum_{n=1}^{\infty} \omega_{n} f_{n}(z)
$$

where $\omega_{n} \geq 0(n \geq 1)$ and $\sum_{n=1}^{\infty} \omega_{n}=1$.

Theorem 9. (Arithmetic Mean:) Let the functions $f_{j}(j=1,2, \ldots m)$ defined by

$$
f_{j}(z)=z-\sum_{n=2}^{\infty} a_{n, j} z^{n} \text { for } a_{n, j} \geq 0, z \in \mathbf{U} .
$$

be in the classes $\mathcal{T} \mathbf{J}_{\mu}^{\eta}\left(\alpha_{j}, \beta, \gamma, A, B\right)(j=1,2, \ldots m)$ respectively. Then the function $h(z)$ defined by

$$
h(z)=z-\frac{1}{m} \sum_{n=2}^{\infty}\left(\sum_{j=1}^{m} a_{n, j}\right)^{z^{n}}
$$

is in the class $\mathcal{T} \mathcal{J}_{\mu}^{\eta}(\alpha, \beta, \gamma, A, B)$, where $\alpha=\min _{1 \leq j \leq m}\left\{\alpha_{j}\right\}, 0 \leq \alpha_{j} \leq 1$.

\section{Holder's Inequality}

Followed by Nishiwaki et al.[11] and Murugusundaramoorthy et al.[10] in this section we study some results of Holder type inequalities for $f \in \mathcal{T} \mathbf{J}_{\mu}^{\eta}(\alpha, \beta, \gamma, A$, $B)$. Now we recall the generalisation of the convolution due to Cho et al.[5] as given below

$$
\mathcal{H}_{m}(z)=z-\sum_{n=2}^{\infty}\left(\prod_{j=1}^{m} a_{n, j}^{p_{j}}\right) z^{n}, \quad\left(p_{j}>0, j=1,2, \ldots m\right) .
$$


Further for functions $f_{j} \in \mathcal{T} \mathbf{J}_{\mu}^{\eta}(\alpha, \beta, \gamma, A, B),(j=1,2 \ldots m)$ given by the familiar Holder inequality assumes the following form

$$
\begin{aligned}
& \sum_{n=2}^{\infty}\left(\prod_{j=1}^{m} a_{n, j}\right) \leq \prod_{j=1}^{m}\left(\sum_{n=2}^{\infty} a_{n, j}^{p_{j}}\right)^{\frac{1}{p_{j}}}, \\
& \left(p_{j}>1, j=1,2 \ldots m, \sum_{j=1}^{m} \frac{1}{p_{j}} \geq 1\right) .
\end{aligned}
$$

Theorem 10. If $f_{j} \in \mathcal{T} \mathbf{J}_{\mu}^{\eta}(\alpha, \beta, \gamma, A, B),-1 \leq B<A \leq 1,0 \leq \alpha<1$ $0<\beta \leq 1,(j=1,2, \ldots m)$ then $\mathcal{H}_{m}(z) \in \mathcal{T} \mathbf{J}_{\mu}^{\eta}(\alpha, \beta, \gamma, A, B)$ with $\xi \leq 1-$

$$
\frac{(2 \beta \gamma(B-A))^{s} \prod_{j=1}^{s}\left(1-\xi_{j}\right)^{p_{j}}(1-(1-B \beta) 2 \beta \gamma(B-A))}{\prod_{j=1}^{m}\left[2 \beta \gamma(B-A)\left(2-\xi_{j}\right)+(1-B \beta)\right]^{p_{j}}\left[c_{2}(\eta, \mu)\right]^{p_{j}-1}-[2 \beta \gamma(B-A)]^{s} \prod_{j=1}^{m}\left(1-\xi_{j}\right)^{p_{j}}},
$$

where

$$
s=\sum_{j=1}^{m} p_{j}>1 ; p_{j} \geq \frac{1}{q_{j}}(j=1,2,3 \ldots m), q_{j}>1(j=1,2 \ldots m) ; \sum_{j=1}^{m} q_{j} \geq 1 .
$$

Proof. Let $f_{j} \in \mathcal{T}^{\eta} \mathcal{J}_{\mu}(\alpha, \beta, \gamma, A, B),(j=1,2 \ldots m)$ then we have

$$
\sum_{n=2}^{\infty} \frac{\left[2 \beta \gamma(B-A)\left(n-\xi_{j}\right)+(1-B \beta)(n-1)\right] c_{n}(\eta, \mu)}{\left.2 \beta \gamma\left(1-\xi_{j}\right)(B-A)\right)} a_{n, j} \leq 1
$$

which in turn implies that

$$
\begin{gathered}
\left(\sum_{n=2}^{\infty} \frac{\left[2 \beta \gamma(B-A)\left(n-\xi_{j}\right)+(1-B \beta)(n-1)\right] c_{n}(\eta, \mu)}{2 \beta \gamma\left(1-\xi_{j}\right)(B-A)} a_{n, j}\right)^{\frac{1}{q_{j}}} \leq 1 \\
\left(q_{j}>1,(j=1,2,3 \ldots m), \sum_{j=1}^{m} \frac{1}{q_{j}}=1\right) .
\end{gathered}
$$

Applying the inequality (27) we arrive at the following inequality

$$
\sum_{n=2}^{\infty}\left(\sum_{j=1}^{m} \frac{\left[2 \beta \gamma(B-A)\left(n-\xi_{j}\right)+(1-B \beta)(n-1)\right]}{2 \beta \gamma\left(1-\xi_{j}\right)(B-A)} c_{n}(\eta, \mu) a_{n, j}\right)^{\frac{1}{q_{j}}} a_{n, j}^{\frac{1}{q_{j}}} \leq 1 .
$$


Thus we determine the largest $\xi$ such that

$$
\left(\sum_{n=2}^{\infty} \frac{\left[2 \beta \gamma(B-A)\left(n-\xi_{j}\right)+(1-B \beta)(n-1)\right] c_{n}(\eta, \mu)}{2 \beta \gamma\left(1-\xi_{j}\right)(B-A)} c_{n}(\eta, \mu)\right) \prod_{j=1}^{m} a_{n, j}^{p_{j}} \leq 1 .
$$

That is

$$
\begin{aligned}
& \left(\sum_{n=2}^{\infty} \frac{\left[2 \beta \gamma(B-A)\left(n-\xi_{j}\right)+(1-B \beta)(n-1)\right] c_{n}(\eta, \mu)}{2 \beta \gamma\left(1-\xi_{j}\right)(B-A)} c_{n}(\eta, \mu)\right) \prod_{j=1}^{m} a_{n, j}^{p_{j}} \leq \\
& \sum_{n=2}^{\infty}\left[\left(\sum_{j=1}^{m} \frac{\left[2 \beta \gamma(B-A)\left(n-\xi_{j}\right)+(1-B \beta)(n-1)\right]}{2 \beta \gamma\left(1-\xi_{j}\right)(B-A)} c_{n}(\eta, \mu) a_{n, j}\right)^{\frac{1}{q_{j}}} a_{n, j}^{\frac{1}{q_{j}}} .\right.
\end{aligned}
$$

Since

$$
\begin{gathered}
\prod_{j=1}^{m}(\frac{\left[2 \beta \gamma(B-A)\left(n-\xi_{j}\right)+(1-B \beta)(n-1)\right]}{2 \beta \gamma\left(1-\xi_{j}\right)(B-A)} c_{n}(\eta, \mu) \underbrace{p_{j}-\frac{1}{q_{j}}} a_{n, j}^{p_{j}-\frac{1}{q_{j}}} \leq 1, \\
\left(p_{j}-\frac{1}{q_{j}} \geq 0, j=1,2,3 \ldots m\right) .
\end{gathered}
$$

We see that

$$
\prod_{j=1}^{m} a_{n, j}^{p_{j}-\frac{1}{q_{j}}} \leq \frac{1}{\prod_{j=1}^{m}\left(\frac{\left[2 \beta \gamma(B-A)\left(n-\xi_{j}\right)+(1-B \beta)(n-1)\right]}{2 \beta \gamma\left(1-\xi_{j}\right)(B-A)} c_{n}(\eta, \mu) p_{j}-\frac{1}{q_{j}}\right.}
$$

This last inequality (28) implies that

$$
\begin{aligned}
& 2 \beta \gamma(B-A) \prod_{j=1}^{m}(2 \beta \gamma(B-A))^{p_{j}-1}\left(1-\xi_{j}\right)^{p_{j}-1} \\
& -\sum_{j=1}^{m}\left[2 \beta \gamma\left(n-\xi_{j}\right)(B-A)+(1-B \beta)(n-1)\right]^{p_{j}}\left(c_{n}(\eta, \mu)\right)^{p_{j}-1}(1-\xi)
\end{aligned}
$$


HOLDER AND INTEGRAL MEANS INEQUALITIES...

$$
\begin{aligned}
& \leq\left(-(n-1)(1-B \beta) \prod_{j=1}^{m}(2 \beta \gamma(B-A))^{p_{j}-1}\left(1-\xi_{j}\right)^{p_{j}}\right) \\
& +\left((n-1) 2 \beta \gamma(B-A) \prod_{j=1}^{m}(2 \beta \gamma(B-A))^{p_{j}-1}\left(1-\xi_{j}\right)^{p_{j}}\right),
\end{aligned}
$$

where

$$
\Upsilon_{j}=\prod_{j=1}^{m}(2 \beta \gamma(B-A))^{p_{j}}\left(1-\xi_{j}\right)^{p_{j}}
$$

Which implies

$$
\begin{gathered}
{\left[\Upsilon_{j}-\sum_{j=1}^{m}\left[2 \beta \gamma\left(n-\xi_{j}\right)(B-A)+(1-B \beta)(n-1)\right]^{p_{j}}\left(c_{n}(\eta, \mu)\right)^{p_{j}-1}(1-\xi) \leq\right.} \\
-\left[(n-1) \Upsilon_{j}+(1-B \beta)(n-1) \prod_{j=1}^{m}(2 \beta \gamma(B-A))^{p_{j}-1}\left(1-\xi_{j}\right)^{p_{j}}\right.
\end{gathered}
$$

That is

$$
\xi \leq 1-\frac{\left[(n-1) \Upsilon_{j}+(1-B \beta)(n-1) \prod_{j=1}^{m}(2 \beta \gamma(B-A))^{p_{j}-1}\left(1-\xi_{j}\right)^{p_{j}}\right]}{\sum_{j=1}^{m}\left[2 \beta \gamma\left(n-\xi_{j}\right)(B-A)+(1-B \beta)(n-1)\right]^{p_{j}}-\Upsilon_{j}} .
$$

Let

$$
\Phi(n) \leq 1-\frac{\left[(n-1) \Upsilon_{j}+(1-B \beta)(n-1) \prod_{j=1}^{m}(2 \beta \gamma(B-A))^{p_{j}-1}\left(1-\xi_{j}\right)^{p_{j}}\right]}{\sum_{j=1}^{m}\left[2 \beta \gamma\left(n-\xi_{j}\right)(B-A)+(1-B \beta)(n-1)\right]^{p_{j}}-\Upsilon_{j}},
$$

which is an increasing function in $n$ hence we have

$$
\begin{aligned}
& \xi \leq \Phi(2)=1- \\
& \frac{(2 \beta \gamma(B-A))^{s} \prod_{j=1}^{s}\left(1-\xi_{j}\right)^{p_{j}}(1-(1-B \beta) 2 \beta \gamma(B-A))}{\prod_{j=1}^{m}\left[2 \beta \gamma(B-A)\left(2-\xi_{j}\right)+(1-B \beta)\right]^{p_{j}}\left[c_{2}(\eta, \mu)\right]^{p_{j}-1}-[2 \beta \gamma(B-A)]^{s} \prod_{j=1}^{m}\left(1-\xi_{j}\right)^{p_{j}}} .
\end{aligned}
$$


Hence the proof.

\section{Integral Means Inequalities}

In this section, we obtain integral means inequalities for the functions in the family $\mathcal{T} \mathbf{J}_{\mu}^{\eta}(\alpha, \beta, \gamma, A, B)$ due to Silverman[20].

Lemma 11. (Littlewood[9])If the functions $f$ and $g$ are analytic in $\mathbf{U}$ with $g \prec f$, then for $\eta>0$, and $0<r<1$,

$$
\int_{0}^{2 \pi}\left|g\left(r e^{i \theta}\right)\right|^{\eta} d \theta \leq \int_{0}^{2 \pi}\left|f\left(r e^{i \theta}\right)\right|^{\eta} d \theta .
$$

In 1975, Silverman[18], found that the function $f_{2}(z)=z-\frac{z^{2}}{2}$ is often extremal over the family $\mathcal{T}$ and applied this function to resolve his integral means inequality, conjectured in Silverman[19] and settled in Silverman [20], that

$$
\int_{0}^{2 \pi}\left|f\left(r e^{i \theta}\right)\right|^{\eta} d \theta \leq \int_{0}^{2 \pi}\left|f_{2}\left(r e^{i \theta}\right)\right|^{\eta} d \theta
$$

for all $f \in \mathcal{T}, \eta>0$ and $0<r<1$. In Silverman[20], also proved his conjecture for the subclasses $\mathcal{T}^{*}(\gamma)$ and $\mathcal{C}(\gamma)$ of $\mathcal{T}$.

Applying Lemma 11, Theorem 6 and Theorem 8, we prove the following result.

Theorem 12. Suppose $f \in \mathcal{T}_{\mathcal{J}}^{\eta}(\alpha, \beta, \gamma, A, B), \eta>0,0 \leq \lambda<1$, $0 \leq \gamma<1, \beta \geq 0$ and $f_{2}(z)$ is defined by

$$
f_{2}(z)=z-\frac{2 \beta \gamma(1-\alpha)(B-A)}{\Phi_{2}(\alpha, \beta, \gamma, A, B)} z^{2},
$$

where $\Phi_{2}(\alpha, \beta, \gamma, A, B)$ is given by $(22)$, and $C_{2}(b, \mu)$ is given by (17). Then for $z=r e^{i \theta}, 0<r<1$, we have

$$
\int_{0}^{2 \pi}|f(z)|^{\eta} d \theta \leq \int_{0}^{2 \pi}\left|f_{2}(z)\right|^{\eta} d \theta .
$$


Proof. For given $f$ of the form (2), from (30) is equivalent to proving that

$$
\int_{0}^{2 \pi}\left|1-\sum_{n=2}^{\infty}\right| a_{n}\left|z^{n-1}\right|^{\eta} d \theta \leq \int_{0}^{2 \pi}\left|1-\frac{(1-\gamma)}{\Phi(\lambda, \gamma, \beta, A, B)} z\right|^{\eta} d \theta .
$$

By Lemma 11, it suffices to show that

$$
1-\sum_{n=2}^{\infty}\left|a_{n}\right| z^{n-1} \prec 1-\frac{2 \beta \gamma(1-\alpha)(B-A)}{\Phi_{2}(\alpha, \beta, \gamma, A, B)} z .
$$

Setting

$$
1-\sum_{n=2}^{\infty}\left|a_{n}\right| z^{n-1}=1-\frac{2 \beta \gamma(1-\alpha)(B-A)}{\Phi_{2}(\alpha, \beta, \gamma, A, B)} w(z)
$$

and using (18), we obtain

$$
\begin{aligned}
|w(z)| & =\left|\sum_{n=2}^{\infty} \frac{\Phi_{n}(\alpha, \beta, \gamma, A, B}{2 \beta \gamma(1-\alpha)(B-A)}\right| a_{n}\left|z^{n-1}\right| \\
& \leq|z| \sum_{n=2}^{\infty} \frac{\Phi_{n}(\alpha, \beta, \gamma, A, B}{2 \beta \gamma(1-\alpha)(B-A)}\left|a_{n}\right| \\
& \leq|z|,
\end{aligned}
$$

where $\Phi_{n}(\alpha, \beta, \gamma, A, B)$ is given by (21).

This completes the proof by Theorem 12 .

\section{Subordination Results}

In this section we obtain subordination results for the new class $\mathcal{T} \mathbf{J}_{\mu}^{\eta}(\alpha, \beta, \gamma, A$, $B)$ due to Wilf [23].

Definition 13. (Subordinating Factor Sequence) A sequence $\left\{b_{n}\right\}_{n=1}^{\infty}$ of complex numbers is said to be a subordinating sequence if, whenever $f(z)=$ $\sum_{n=1}^{\infty} a_{n} z^{n}, a_{1}=1$ is regular, univalent and convex in $\mathbf{U}$, we have

$$
\sum_{n=1}^{\infty} b_{n} a_{n} z^{n} \prec f(z), \quad z \in \mathbf{U}
$$


Lemma 14. (Wilf, [23]) The sequence $\left\{b_{n}\right\}_{n=1}^{\infty}$ is a subordinating factor sequence if and only if

$$
\Re\left\{1+2 \sum_{n=1}^{\infty} b_{n} z^{n}\right\}>0, \quad z \in \mathbf{U} .
$$

For the sake of brevity, we let

$$
\psi_{n}(\alpha, \beta, \gamma, A, B)=\Phi_{n}(\alpha, \beta, \gamma, A, B) C_{n}(\eta, \mu)
$$

and

$$
\psi_{2}(\alpha, \beta, \gamma, A, B)=\Phi_{2}(\alpha, \beta, \gamma, A, B) C_{2}(\eta, \mu)
$$

Theorem 15. Let $f \in \mathcal{T} \mathbf{J}_{\mu}^{\eta}(\alpha, \beta, \gamma, A, B)$ and $g(z)$ be any function in the usual class of convex functions $\mathcal{C} \mathcal{V}$, then

$$
\frac{\psi_{2}(\alpha, \beta, \gamma, A, B)}{2\left[2 \beta \gamma(1-\alpha)(B-A)+\psi_{2}(\alpha, \beta, \gamma, A, B)\right]}(f * g)(z) \prec g(z),
$$

where $0 \leq \gamma<1 ; \beta \geq 0$ and $0 \leq \lambda<1$, and

$$
\Re(f(z))>-\frac{\left[2 \beta \gamma(1-\alpha)(B-A)+\psi_{2}(\alpha, \beta, \gamma, A, B)\right]}{\psi_{2}(\alpha, \beta, \gamma, A, B)}, z \in \mathbf{U} .
$$

The constant factor $\frac{\left[2 \beta \gamma(1-\alpha)(B-A)+\psi_{2}(\alpha, \beta, \gamma, A, B)\right]}{\psi_{2}(\alpha, \beta, \gamma, A, B)}$ in (36) cannot be replaced by a larger number.

Proof. Let $f \in \mathcal{T} \mathbf{J}_{\mu}^{\eta}(\alpha, \beta, \gamma, A, B)$ and suppose that $g(z)=z+\sum_{n=2}^{\infty} b_{n} z^{n} \in \mathbf{C}$. Then

$$
\begin{aligned}
& \frac{\psi_{2}(\alpha, \beta, \gamma, A, B)}{2\left[2 \beta \gamma(1-\alpha)(B-A)+\psi_{2}(\alpha, \beta, \gamma, A, B)\right]}(f * g)(z) \\
& \quad=\frac{\psi_{2}(\alpha, \beta, \gamma, A, B)}{2\left[2 \beta \gamma(1-\alpha)(B-A)+\psi_{2}(\alpha, \beta, \gamma, A, B)\right]}\left(z+\sum_{n=2}^{\infty} b_{n} a_{n} z^{n}\right) .
\end{aligned}
$$

Thus, by Definition 13, the subordination result holds true if

$$
\left\{\frac{\psi_{2}(\alpha, \beta, \gamma, A, B)}{2\left[2 \beta \gamma(1-\alpha)(B-A)+\psi_{2}(\alpha, \beta, \gamma, A, B)\right]}\right\}_{n=1}^{\infty}
$$


is a subordinating factor sequence, with $a_{1}=1$. In view of Lemma 14, this is equivalent to the following inequality

$$
\Re\left\{1+\sum_{n=1}^{\infty} \frac{\psi_{2}(\alpha, \beta, \gamma, A, B)}{2\left[2 \beta \gamma(1-\alpha)(B-A)+\psi_{2}(\alpha, \beta, \gamma, A, B)\right]} a_{n} z^{n}\right\}>0, \quad z \in \mathbf{U} .
$$

By noting the fact that $\psi_{n}(\alpha, \beta, \gamma, A, B)$ is increasing function for $n \geq 2$ and in particular

$$
\frac{\psi_{2}(\alpha, \beta, \gamma, A, B)}{2 \beta \gamma(1-\alpha)(B-A)} \leq \frac{\psi_{n}(\alpha, \beta, \gamma, A, B)}{2 \beta \gamma(1-\alpha)(B-A)}, \quad n \geq 2,
$$

therefore, for $|z|=r<1$, we have

$$
\begin{array}{ll} 
& \Re\left\{1+\frac{\psi_{2}(\alpha, \beta, \gamma, A, B)}{2\left[2 \beta \gamma(1-\alpha)(B-A)+\psi_{2}(\alpha, \beta, \gamma, A, B)\right]} \sum_{n=1}^{\infty} a_{n} z^{n}\right\} \\
=\quad \Re\left\{1+\frac{\psi_{2}(\alpha, \beta, \gamma, A, B)}{2\left[2 \beta \gamma(1-\alpha)(B-A)+\psi_{2}(\alpha, \beta, \gamma, A, B)\right]} z\right. & \left.\quad \frac{\psi_{2}(\alpha, \beta, \gamma, A, B)}{2\left[2 \beta \gamma(1-\alpha)(B-A)+\psi_{2}(\alpha, \beta, \gamma, A, B)\right]} a_{n} z^{n}\right\} \\
\geq \quad & 1-\frac{\psi_{2}(\alpha, \beta, \gamma, A, B)}{2\left[2 \beta \gamma(1-\alpha)(B-A)+\psi_{2}(\alpha, \beta, \gamma, A, B)\right]} r \\
-\quad & \frac{1}{\left[2 \beta \gamma(1-\alpha)(B-A) \psi_{2}(\alpha, \beta, \gamma, A, B)\right]} \\
\sum_{n=2}^{\infty} \quad & \left|[2 \beta \gamma(B-A)(n-\alpha)+(1-B \beta)(n-1)] C_{n}(\eta, \mu) a_{n}\right| r^{n} \\
\geq \quad & 1-\frac{\psi_{2}(\alpha, \beta, \gamma, A, B)}{2\left[2 \beta \gamma(1-\alpha)(B-A)+\psi_{2}(\alpha, \beta, \gamma, A, B)\right]} r \\
-\quad & \frac{2 \beta \gamma(1-\alpha)(B-A)}{\left[2 \beta \gamma(1-\alpha)(B-A)+\psi_{2}(\alpha, \beta, \gamma, A, B)\right]} r>0,(|z|=r),
\end{array}
$$

where we have also made use of the assertion (18) of Theorem 6 . This evidently proves the inequality (39) and hence also the subordination result (36) asserted by Theorem 15 . The inequality (37) follows from (36) by taking

$$
g(z)=\frac{z}{1-z}=z+\sum_{n=2}^{\infty} z^{n} \in \mathbf{C} .
$$

Next we consider the function

$$
F(z):=z-\frac{2 \beta \gamma(1-\alpha)(B-A)}{\psi_{2}(\alpha, \beta, \gamma, A, B)} z^{2}
$$


where $0 \leq \gamma<1, \beta \geq 0,0 \leq \lambda<1$. Clearly $F \in \mathcal{T} \mathbf{J}_{\mu}^{\eta}(\rho, \alpha, \beta, \gamma, A, B)$. For this function (36) becomes

$$
\frac{\psi_{2}(\alpha, \beta, \gamma, A, B)}{2\left[2 \beta \gamma(1-\alpha)(B-A)+\psi_{2}(\alpha, \beta, \gamma, A, B)\right]} F(z) \prec \frac{z}{1-z} .
$$

It is easily verified that

$$
\min \left\{\Re\left(\frac{\psi_{2}(\alpha, \beta, \gamma, A, B)}{2\left[2 \beta \gamma(1-\alpha)(B-A)+\psi_{2}(\alpha, \beta, \gamma, A, B)\right]} F(z)\right)\right\}=-\frac{1}{2}, \quad z \in \mathbf{U} .
$$

This shows that the constant

$$
\frac{\psi_{2}(\alpha, \beta, \gamma, A, B)}{2\left[2 \beta \gamma(1-\alpha)(B-A)+\psi_{2}(\alpha, \beta, \gamma, A, B)\right]}
$$

cannot be replaced by any larger one.

\section{Convex Linear Combination}

In the following theorem, we show that the classes $\mathcal{T} \mathbf{J}_{\mu}^{\eta}(\alpha, \beta, \gamma, A, B)$ are closed under convex linear combination.

Theorem 16. The class $\mathcal{T} \mathbf{J}_{\mu}^{\eta}(\alpha, \beta, \gamma, A, B)$ is closed under convex linear combination.

Proof. We want to show the function

$$
F(z)=(1-\mu) f_{1}(z)+\mu f_{2}(z), 0 \leq \mu \leq 1
$$

is in the class $\mathcal{T} \mathbf{J}_{\mu}^{\eta}(\alpha, \beta, \gamma, A, B)$, where $f_{1}, f_{2} \in \mathcal{T} \mathbf{J}_{\mu}^{\eta}(\alpha, \beta, \gamma, A, B)$ and

$$
f_{1}(z)=z-\sum_{n=2}^{\infty} a_{n, 1} z^{n}, f_{2}(z)=z-\sum_{n=2}^{\infty} a_{n, 2} z^{n} .
$$

By (18) we have

$$
\sum_{n=2}^{\infty} \Phi_{n}(\alpha, \beta, \gamma, A, B) C_{n}(\eta, \mu) a_{n, 1} \leq 2 \beta \gamma(1-\alpha)(B-A)
$$

and

$$
\sum_{n=2}^{\infty} \Phi_{n}(\alpha, \beta, \gamma, A, B) C_{n}(\eta, \mu) a_{n, 2} \leq 2 \beta \gamma(1-\alpha)(B-A)
$$


Therefore

$$
\begin{aligned}
F(z) & =(1-\mu) f_{1}(z)+\mu f_{2}(z) \\
& =(1-\mu)\left(z-\sum_{n=2}^{\infty} a_{n, 1} z^{n}\right)+\mu\left(z-\sum_{n=2}^{\infty} a_{n, 2} z^{n}\right) \\
& =z-\sum_{n=2}^{\infty}\left((1-\mu) a_{n, 1}+\mu a_{n, 2}\right) z^{n} .
\end{aligned}
$$

We must show that $F(z)$ with the coefficient $\left((1-\mu) a_{n, 1}+\mu a_{n, 2}\right)$ satisfy in the relation (18) also the coefficient $\left((1-\mu) a_{n, 1}+\mu a_{n, 2}\right)$ satisfy in the inequality in Corollary 7. Further

$$
\begin{aligned}
& \sum_{n=2}^{\infty}[n-(1+n \lambda-\lambda) \alpha]\left((1-\mu) a_{n, 1}+\mu a_{n, 2}\right) \frac{(a)_{n-1}(b)_{n-1}}{(c)_{n-1}(1)_{n-1}} \\
= & (1-\mu) \sum_{n=2}^{\infty}[n-(1+n \lambda-\lambda) \alpha] \frac{(a)_{n-1}(b)_{n-1}}{(c)_{n-1}(1)_{n-1}} a_{n, 1} \\
& \quad+\mu \sum_{n=2}^{\infty}[n-(1+n \lambda-\lambda) \alpha] \frac{(a)_{n-1}(b)_{n-1}}{(c)_{n-1}(1)_{n-1}} a_{n, 2} \\
\leq & (1-\mu)(\alpha-1)+\mu(\alpha-1) \\
= & \alpha-1 .
\end{aligned}
$$

Therefore, it follows that $F \in \mathcal{T} \mathbf{J}_{\mu}^{\eta}(\alpha, \beta, \gamma, A, B)$

\section{Partial Sum Results}

Silvia [21] studied the partial sums of convex functions of order $\alpha$. Later on, Abubaker and Darus [2], Frasin [6], Raina and Bansal [14] and Rosy et al.[15] determined the sharp lower bound on the real part of the quotients between the normalized starlike or convex functions, viz., $\Re\left(\frac{f(z)}{f_{k}(z)}\right), \Re\left(\frac{f_{k}(z)}{f(z)}\right), \Re\left(\frac{f^{\prime}(z)}{f_{k}^{\prime}(z)}\right)$ and $\Re\left(\frac{f_{k}^{\prime}(z)}{f^{\prime}(z)}\right)$ for their sequences of partial sums $f_{k}(z)=z+\sum_{n=2}^{k} a_{n} z^{n}$ of the analytic function $f(z)=z+\sum_{n=2}^{\infty} a_{n} z^{n}$.

In the following theorems we discuss the results on partial sums for $f \in \mathcal{T} \mathbf{J}_{\mu}^{\eta}(\alpha, \beta$, $\gamma, A, B)$. 
Theorem 17. If $f$ of the form (1) satisfies the condition (18), then

$$
\Re\left(\frac{f(z)}{f_{k}(z)}\right) \geq \frac{\rho_{k+1}(\alpha, \beta, \gamma, A, B)-2 \beta \gamma(1-\alpha)(B-A)}{\rho_{k+1}(\alpha, \beta, \gamma, A, B)}, \quad(z \in \mathbf{U}),
$$

where

$$
\rho_{n}=\rho_{k+1}(\alpha, \beta, \gamma, A, B) \geq\left\{\begin{array}{l}
2 \beta \gamma(1-\alpha)(B-A), \quad \text { if } n=2,3, \ldots, k \\
\rho_{k+1}, \quad \text { if } n=k+1, k+2, \ldots .
\end{array}\right.
$$

The result (41) is sharp with the function given by

$$
f(z)=z+\frac{2 \beta \gamma(1-\alpha)(B-A)}{\rho_{k+1}} z^{n+1} .
$$

Proof. Define the function $w(z)$ by

$$
\begin{aligned}
\frac{1+w(z)}{1-w(z)} & =\frac{\rho_{k+1}}{2 \beta \gamma(1-\alpha)(B-A)}\left[\frac{f(z)}{f_{k}(z)}-\frac{\rho_{k+1}-2 \beta \gamma(1-\alpha)(B-A)}{\rho_{k+1}}\right] \\
& =\frac{1+\sum_{n=2}^{k} a_{n} z^{n-1}+\left(\frac{\rho_{k+1}}{2 \beta \gamma(1-\alpha)(B-A)}\right) \sum_{n=k+1}^{\infty} a_{n} z^{n-1}}{1+\sum_{n=2}^{k} a_{n} z^{n-1}}
\end{aligned}
$$

It suffices to show that $|w(z)| \leq 1$. Now, from (44) we can write

$$
w(z)=\frac{\left(\frac{\rho_{k+1}}{2 \beta \gamma(1-\alpha)(B-A)}\right) \sum_{n=k+1}^{\infty} a_{n} z^{n-1}}{2+2 \sum_{n=2}^{k} a_{n} z^{n-1}+\left(\frac{\rho_{k+1}}{2 \beta \gamma(1-\alpha)(B-A)}\right) \sum_{n=k+1}^{\infty} a_{n} z^{n-1}} .
$$

Hence we obtain

$$
|w(z)| \leq \frac{\left(\frac{\rho_{k+1}}{2 \beta \gamma(1-\alpha)(B-A)}\right) \sum_{n=k+1}^{\infty}\left|a_{n}\right|}{2-2 \sum_{n=2}^{k}\left|a_{n}\right|-\left(\frac{\rho_{k+1}}{2 \beta \gamma(1-\alpha)(B-A)}\right) \sum_{n=k+1}^{\infty}\left|a_{n}\right|} .
$$

Now $|w(z)| \leq 1$, if and only if

$$
2\left(\frac{\rho_{k+1}}{2 \beta \gamma(1-\alpha)(B-A)}\right) \sum_{n=k+1}^{\infty}\left|a_{n}\right| \leq 2-2 \sum_{n=2}^{k}\left|a_{n}\right|
$$


Or, equivalently,

$$
\sum_{n=2}^{k}\left|a_{n}\right|+\sum_{n=k+1}^{\infty} \frac{\rho_{k+1}}{2 \beta \gamma(1-\alpha)(B-A)}\left|a_{n}\right| \leq 1
$$

From the condition (18), it is sufficient to show that

$$
\sum_{n=2}^{k}\left|a_{n}\right|+\sum_{n=k+1}^{\infty} \frac{\rho_{k+1}}{2 \beta \gamma(1-\alpha)(B-A)}\left|a_{n}\right| \leq \sum_{n=2}^{\infty} \frac{\rho_{n}}{2 \beta \gamma(1-\alpha)(B-A)}\left|a_{n}\right|
$$

which is equivalent to

$$
\begin{aligned}
& \sum_{n=2}^{k}\left(\frac{\rho_{n}-2 \beta \gamma(1-\alpha)(B-A)}{2 \beta \gamma(1-\alpha)(B-A)}\right)\left|a_{n}\right| \\
+ & \sum_{n=k+1}^{\infty}\left(\frac{\rho_{n}-\rho_{k+1}}{2 \beta \gamma(1-\alpha)(B-A)}\right)\left|a_{n}\right| \geq 0 .
\end{aligned}
$$

To see that the function given by (43) gives the sharp result, we observe that for $z=r e^{i \pi / k}$

$$
\begin{aligned}
\frac{f(z)}{f_{k}(z)} & =1+\frac{2 \beta \gamma(1-\alpha)(B-A)}{\rho_{k+1}} z^{k} \rightarrow 1-\frac{2 \beta \gamma(1-\alpha)(B-A)}{\rho_{k+1}} \\
& =\frac{\rho_{k+1}-2 \beta \gamma(1-\alpha)(B-A)}{\rho_{k+1}} \text { when } r \rightarrow 1^{-} .
\end{aligned}
$$

We next determine bounds for $\Re\left(f_{k}(z) / f(z)\right)$.

Theorem 18. If $f$ of the form (1) satisfies the condition (18), then

$$
\Re\left(\frac{f_{k}(z)}{f(z)}\right) \geq \frac{\rho_{k+1}}{\rho_{k+1}+2 \beta \gamma(1-\alpha)(B-A)}, \quad(z \in \mathbf{U}),
$$

where $\rho_{k+1} \geq 2 \beta \gamma(1-\alpha)(B-A)$ and

$$
\left.\rho_{n}(\lambda, \gamma, \eta)\right) \geq\left\{\begin{array}{l}
2 \beta \gamma(1-\alpha)(B-A), \\
\rho_{k+1}, \quad \text { if } n=k+1, k+2, \ldots
\end{array} \quad \text { if } n=2,3, \ldots, k\right.
$$

The result (46) is sharp with the function given by (43). 
Proof. We write

$$
\begin{gathered}
\frac{1+w(z)}{1-w(z)}=\frac{\rho_{k+1}+2 \beta \gamma(1-\alpha)(B-A)}{2 \beta \gamma(1-\alpha)(B-A)}\left[\frac{f_{k}(z)}{f(z)}-\frac{\rho_{k+1}}{\rho_{k+1}+2 \beta \gamma(1-\alpha)(B-A)}\right] \\
=\frac{1+\sum_{n=2}^{k} a_{n} z^{n-1}-\left(\frac{\rho_{k+1}}{2 \beta \gamma(1-\alpha)(B-A)}\right) \sum_{n=k+1}^{\infty} a_{n} z^{n-1}}{1+\sum_{n=2}^{\infty} a_{n} z^{n-1}}
\end{gathered}
$$

where

$$
|w(z)| \leq \frac{\left(\frac{\rho_{k+1}+2 \beta \gamma(1-\alpha)(B-A)}{2 \beta \gamma(1-\alpha)(B-A)}\right) \sum_{n=k+1}^{\infty}\left|a_{n}\right|}{2-2 \sum_{n=2}^{k}\left|a_{n}\right|-\left(\frac{\rho_{k+1}-2 \beta \gamma(1-\alpha)(B-A)}{2 \beta \gamma(1-\alpha)(B-A)}\right) \sum_{n=k+1}^{\infty}\left|a_{n}\right|} \leq 1
$$

This last inequality is equivalent to

$$
\sum_{n=2}^{k}\left|a_{n}\right|+\sum_{n=k+1}^{\infty} \frac{\rho_{k+1}}{2 \beta \gamma(1-\alpha)(B-A)}\left|a_{n}\right| \leq 1
$$

Making use of (18) to get (45). Finally, equality holds in (46) for the extremal function $f(z)$ given by $(43)$.

We next turns to ratios involving derivatives.

Theorem 19. If $f$ of the form (1) satisfies the condition (18), then

$$
\Re\left(\frac{f^{\prime}(z)}{f_{k}^{\prime}(z)}\right) \geq \frac{\rho_{k+1}-2 \beta \gamma(k+1)(1-\alpha)(B-A)}{\rho_{k+1}}, \quad(z \in \mathbf{U}),
$$

and

$$
\Re\left(\frac{f_{k}^{\prime}(z)}{f^{\prime}(z)}\right) \geq \frac{\rho_{k+1}}{\rho_{k+1}+2 \beta \gamma(k+1)(1-\alpha)(B-A)}, \quad(z \in \mathbf{U}),
$$

where $\rho_{k+1} \geq 2 \beta \gamma(k+1)(1-\alpha)(B-A)$. and

$$
\rho_{k} \geq\left\{\begin{array}{l}
2 \beta \gamma k(1-\alpha)(B-A), \quad \text { if } n=2,3, \ldots, k \\
n\left(\frac{\rho_{k+1}}{k+1}\right), \quad \text { if } n=k+1, k+2, \ldots
\end{array}\right.
$$

The results are sharp with the function given by (43). 
Proof. We write

$$
\begin{aligned}
& \frac{1+w(z)}{1-w(z)}=\frac{\rho_{k+1}}{2 \beta \gamma(k+1)(1-\alpha)(B-A)} \\
& \times\left[\frac{f^{\prime}(z)}{f_{k}^{\prime}(z)}-\left(\frac{\rho_{k+1}-2 \beta \gamma(k+1)(1-\alpha)(B-A)}{\rho_{k+1}}\right)\right] .
\end{aligned}
$$

where

$$
w(z)=\frac{\left(\frac{\rho_{k+1}}{2 \beta \gamma(k+1)(1-\alpha)(B-A)}\right) \sum_{n=k+1}^{\infty} n a_{n} z^{n-1}}{2+2 \sum_{n=2}^{k} n a_{n} z^{n-1}+\left(\frac{\rho_{k+1}}{2 \beta \gamma(k+1)(1-\alpha)(B-A)}\right) \sum_{n=k+1}^{\infty} n a_{n} z^{n-1}} .
$$

Now $|w(z)| \leq 1$ if and only if

$$
\sum_{n=2}^{k} n\left|a_{n}\right|+\frac{\rho_{k+1}}{2 \beta \gamma(k+1)(1-\alpha)(B-A)} \sum_{n=k+1}^{\infty} n\left|a_{n}\right| \leq 1 .
$$

From the condition (18), it is sufficient to show that

$$
\begin{aligned}
& \sum_{n=2}^{k} n\left|a_{n}\right|+\frac{\rho_{k+1}}{2 \beta \gamma(k+1)(1-\alpha)(B-A)} \sum_{n=k+1}^{\infty} n\left|a_{n}\right| \\
& \quad \leq \sum_{n=2}^{\infty} \frac{\rho_{n}}{2 \beta \gamma(1-\alpha)(B-A)}\left|a_{n}\right|
\end{aligned}
$$

which is equivalent to

$$
\begin{aligned}
& \sum_{n=2}^{k}\left(\frac{\rho_{n}-2 \beta \gamma(1-\alpha)(B-A) n}{2 \beta \gamma(1-\alpha)(B-A)}\right)\left|a_{n}\right|+ \\
& \sum_{n=k+1}^{\infty} \frac{(k+1) \rho_{n}-n \rho_{k+1}}{2 \beta \gamma(k+1)(1-\alpha)(B-A)}\left|a_{n}\right| \geq 0 .
\end{aligned}
$$

To prove the result (49), define the function $w(z)$ by

$$
\begin{aligned}
& \frac{1+w(z)}{1-w(z)}=\frac{(k+1)(2 \beta \gamma(1-\alpha)(B-A))+\rho_{k+1}}{2 \beta \gamma(k+1)(1-\alpha)(B-A)} \\
& \times\left[\frac{f_{k}^{\prime}(z)}{f^{\prime}(z)}-\frac{\rho_{k+1}}{2 \beta \gamma(k+1)(1-\alpha)(B-A)+\rho_{k+1}}\right]
\end{aligned}
$$


where

$$
w(z)=\frac{-\left(1+\frac{\rho_{k+1}}{2 \beta \gamma(k+1)(1-\alpha)(B-A)}\right) \sum_{n=k+1}^{\infty} n a_{n} z^{n-1}}{2+2 \sum_{n=2}^{k} n a_{n} z^{n-1}+\left(1-\frac{\rho_{k+1}}{2 \beta \gamma(k+1)(1-\alpha)(B-A)}\right) \sum_{n=k+1}^{\infty} n a_{n} z^{n-1}} .
$$

Now $|w(z)| \leq 1$ if and only if

$$
\sum_{n=2}^{k} n\left|a_{n}\right|+\left(\frac{\rho_{k+1}}{2 \beta \gamma(k+1)(1-\alpha)(B-A)}\right) \sum_{n=k+1}^{\infty} n\left|a_{n}\right| \leq 1 .
$$

It suffices to show that the left hand side of (51) is bounded above by the condition

$$
\sum_{n=2}^{\infty} \frac{\rho_{n}}{2 \beta \gamma(1-\alpha)(B-A)}\left|a_{n}\right|
$$

which is equivalent to

$$
\begin{array}{r}
\sum_{n=2}^{k}\left(\frac{\rho_{n}}{2 \beta \gamma(1-\alpha)(B-A)}-n\right)\left|a_{n}\right| \\
+\sum_{n=k+1}^{\infty}\left(\frac{\rho_{n}}{2 \beta \gamma(1-\alpha)(B-A)}-\frac{\rho_{k+1}}{2 \beta \gamma(k+1)(1-\alpha)(B-A)}\right) n\left|a_{n}\right| \geq 0 .
\end{array}
$$

Acknowledgement: I express my sincere thanks to my guide Prof. Dr. G. Murugusundaramoorthy for his valuable suggestions and informative remarks for presenting this paper.

\section{References}

[1] Aghalary, R. and S. Kullkarni (2002). Some theorems on univalent functions, J. Indian Acad. Math., 24 (1), pp. 81-93.

[2] Afaf, A. Ali Abubaker and M. Darus Partial sums of analytic functions involving generalized Cho-Kwon-Srivastava operator, Int. J. Open Prob. Compl. Anal., 2(3)(2010)., pp. 181-188.

[3] Bernardi, S. D. Convex and starlike univalent functions, Trans. Amer. Math. Soc., 135 (1969)., pp. 429-446.

[4] Caputo, M. Linear models of dissipation whose $Q$ is almost frequency independent. II, Geophys. J. Roy. Astronom. Soc. 13(1967)., pp. 529-539. 
[5] Cho, N. E., T. H. Kim and S. Owa Generalizations of hadamard products of functions with negative coefficients, J. Math. Anal. Appl., 199(1996), pp. 495-501.

[6] Frasin, B. A. Partial sums of certain analytic and univalent functions, Acta Math. Acad. Paed. Nyir., 21(2005)., pp. 135-145.

[7] Khairanar, S. M. and Meena Certain family of analytic and univalent functions with normalized conditions, Acta Math. Acade. Paeda. Nyire., 24(2008)., pp. 333-344.

[8] R.J. Libera Some classes of regular univalent functions, Proc. Amer. Math. Soc.,(1969), 16, 755-758.

[9] Littlewood, J. E. On inequalities in theory of functions, Proc. London Math. Soc., 23(1925), pp. 481-519.

[10] Murugusundaramoorthy, G., K. Vijaya and K. Deepa Holder inequalities for a subclass of univalent functions involving Dzoik Srivastava operator, Global Journal of Mathematical Analysis., 1(3)(2013), pp. 74-82.

[11] Nishiwaki, J., S. Owa and H. M. Srivastava Convolution and Holder type inequalities for a certain class of analytic functions, Math. Inequal. Appl., 11(2008), pp. 717-727.

[12] Owa, S. and J. Nishiwaki Coefficient estimates for certain classes of analytic functions, J. Inequal. Pure. Appl. Math., 3(5)(2002), Art.72.

[13] Owa, S. Some properties of fractional calculus operators for certain analytic functions, in M.Darus and S. Owa, editors, Proc. Intet nat. Symp. on New Development of Geometric Function Theory and its Applications, (GFTA 2008; November 10-13, 2008), Bangi, Selangor, Malaysia,(2008) pp. 214-221.

[14] Raina, R. K. and Deepak Bansal Some properties of a new class of analytic functions defined in terms of a Hadamard product,(2008). J. Inequl. Pure Appl. Math., 9 (1), Art. 22, pp. $1-20$.

[15] Rosy, T., K. G. Subramanian and G. Murugusundaramoorthy . Neighbourhoods and partial sums of starlike functions based on Ruscheweyh derivatives, J. Ineq. Pure and Appl. Math., 4(64)(2003), pp. 1-8.

[16] Salagean, G. S. Subclasses of univalent functions, Lecture Notes in Math., 1013, SpringerVerlag,(1983) pp. 362-372.

[17] Salah, J. and M. Darus A subclass of uniformly convex functions associated with a fractional calculus operator involving Caputo's fractional differentiation, Acta Universitatis Apulensis. 24(2010), pp. 295-304.

[18] Silverman, H. Univalent functions with negative coefficients, Proc. Amer. Math. Soc., 51(1975), pp. 109-116.

[19] Silverman, H. A survey with open problems on univalent functions whose coefficients are negative, Rocky Mt. J. Math., 21 (1991), pp. 1099-1125.

[20] Silverman, H. Integral means for univalent functions with negative coefficients, Houston J. Math., 23(1997)., pp. 169-174.

[21] Silvia, E. M. On partial Sums of convex functions of order $\alpha$, Houston J. Math., 11 (3)(1985), pp. 397-404.

[22] Srivastava,H.M. and S.Owa. Some characterization and distortion theorems involving fractional calculus, generalized hypergeometric functions, Hadamard products, linear operators and certain subclasses of analytic functions, Nagoya Math. J., 106(1987),pp.128. 
[23] Wilf, H. S. Subordinating factor sequence for convex maps of the unit circle, Proc. Amer. Math. Soc., 12(1961), pp. 689-693. 